\title{
Big-Data-Enabled Software-Defined Cellular Network Management
}

\author{
Jiayao Wen and Victor O.K. Li \\ Department of Electrical and Electronic Engineering \\ The University of Hong Kong, Pokfulam, Hong Kong, China \\ E-mail: \{jywen, vli\}@eee.hku.hk
}

\begin{abstract}
With the development of big data collection and analysis technologies, the abundant mobile network information and traffic data provide opportunities for researchers to analyze and understand mobile networks better. However, the existing cellular network architecture do not facilitate the deployment of big mobile data analysis in practical networks. In order to use these data for network management, in this paper, we propose a new software-defined cellular network (SDCN) architecture, namely Big-Data-Enabled Architecture (BDEA), which can support big mobile data analysis and storage for efficient cellular network resource allocation. Based on BDEA, we also propose a virtuous network management cycle of data collection, data analysis and network deployment, which can increase the efficiency of network improvement and feedback. Several application cases of network resource allocation in BDEA are discussed to illustrate how big mobile data analysis can benefit network optimization.
\end{abstract}

\section{INTRODUCTION}

With the rapid development of cellular networks, the amount of network information and traffic data has exploded in recent years. As the coverage area and the number of devices increase, more information about devices and users needs to be processed and managed. In addition, the exponential growth in data traffic increases the burden on network management. Statistics [1] show that the total mobile data traffic is expected to rise at a compound annual growth rate of around 65 percent.

The abundant mobile network information and traffic data provide opportunities for researchers to analyze and understand mobile networks from different aspects. Efforts have been made to extract the analysis results from abundant mobile data. These results, including user profiles and mobility patterns, allow networks to be operated more efficiently.

However, the existing cellular network architectures do not facilitate the deployment of mobile data analysis in practical networks. For instance, in Long Term Evolution (LTE) networks, the control logic are mainly integrated in base stations (BS), Mobility Management Entity (MME) and gateways (GW). The functionality in these network elements are usually pre-built by vendors and cannot be easily modified by carriers. Besides, limited to the computing capability for big data analysis, today's mobile data analysis are usually performed in remote data centers. The analysis results cannot be applied to network deployment and management directly.

In this paper, we propose a new SDCN architecture supported by a big data storage and analysis platform, known as Big-Data-Enabled Architecture (BDEA). Based on BDEA, we design a virtuous cycle of data collection, data analysis and network deployment for network management. The reason we choose software-defined networking (SDN) for mobile data analysis result deployment is because of its friendly application extension, centralized computing capability and global control view. SDN provides an open protocol to deploy new rules or functions over multiple network switches in a programmable way, simplifying network management and service extension. While SDN is deployed in cellular networks, the central controller can provide greater storage and computing capability to implement data analysis, compared to the BS and MME in traditional networks. In addition, the controller manages the network with a global view, which can maximize resource allocation efficiency by utilizing global data analysis.

In BDEA, the data platform in the control plane uses the ultra-high storage and computing capability to store and analyze network historical data. The data platform can relieve the heavy burden on the controller and provide finegrained network analysis reports. With our proposed network management cycle, the analysis results generated in the data platform, including prediction results of network status, can be used to modify parameters of resource allocation algorithms and then applied to the network through SDN deployment. To summarize, we make the following contributions:

- Design an SDCN architecture with a data platform which supports big data storage and analysis.

- Introduce data exchange and component cooperation in the control plane, which utilizes the computing capability of big data analysis for efficient resource allocation.

- Propose a virtuous management cycle of data collection, data analysis and network deployment based on BDEA.

- Illustrate the potential of using data analysis to improve resource allocation efficiency with application cases.

The rest of this paper is organized as follows. Section II presents the related work. We describe BDEA and the proposed data cycle in Section III. In Section IV and V, we discuss several application cases, challenges and future work. We conclude the paper in Section VI.

\section{BACKGROUND}

In this section, we will introduce the achievement on big mobile data analysis and the related architectures.

\section{A. Scalable mobile data analysis}

Because of the increasing demands on wireless communications, monitoring and analysis of large-scale mobile network 
traffic is of both theoretical and practical significance for optimizing network resource and improving user experience.

Many research efforts have been expended on monitoring, storing and analyzing data in cellular networks. A traffic monitoring and analysis system for large-scale cellular network is presented in [2], which can process 4.2 Tbytes of traffic data from $123 \mathrm{~Gb} / \mathrm{s}$ links with high performance and low cost.

For big data analysis, a Hadoop-based tool is proposed to process packet trace files in parallel [3]. In [4], a detailed analysis of user behavior based on big mobile data is presented. The data volume they collected and analyzed is up to 60.81 TB, covering 4.5 million mobile subscribers and 181 thousand cells in a one-week period.

The analysis results include but are not limited to user behavior on data and service usage [4], mobility prediction [5], and user clustering. However, these analysis results suffer a long time lag before they can feedback onto the cellular network from which the data are collected. The long time lag weakens the effects of network improvement, as the network conditions are changing all the time.

\section{B. SDCN architecture}

SDN abstracts the control plane of networks to a central controller. It enjoys the advantage of flexible and global management. The concept of SDN has been extended to a wide variety of network environments, including cellular networks. Many designs of SDCN architecture have been proposed in the last several years.

Li et al. suggested Cellular SDN in [6]. They proposed four main extensions to the controllers, switches and base stations. They also discussed several controller applications, which are major challenges in cellular networks, but can get better solutions with SDN.

SoftRAN [7] proposes to abstract all the base stations in a geographical area to a virtual big base station. The physical base stations are considered as radio elements with minimal control logic. The resources in the network are described in a three dimensional grid of space, time, and frequency slots.

SoftCell [8] proposes to implement high-level service polices by directing traffic through sequences of middleboxs based on subscriber attributes and applications. Traffic in SoftCell is aggregated along multiple dimensions, which can reduce the size of switch tables significantly.

SoftMoW [9] builds a hierarchical and reconfigurable network-wide control plane. It uses a label-swapping mechanism to implement end-to-end paths while keeping per packet overhead minimal.

However, the architectures above do not discuss big data storage and analysis in the control plane. With unprecedented centralized control logic and computing capability, SDCN has great potential to allocate network resources efficiently by exploiting the massive amount of global mobile data.

\section{PROPOSED NETWORK MANAGEMENT MODEL}

In this section, we will firstly give an overview of BDEA. Then the components and data exchanges in the control plane will be introduced. Finally, we will describe the virtuous cycle of network management.

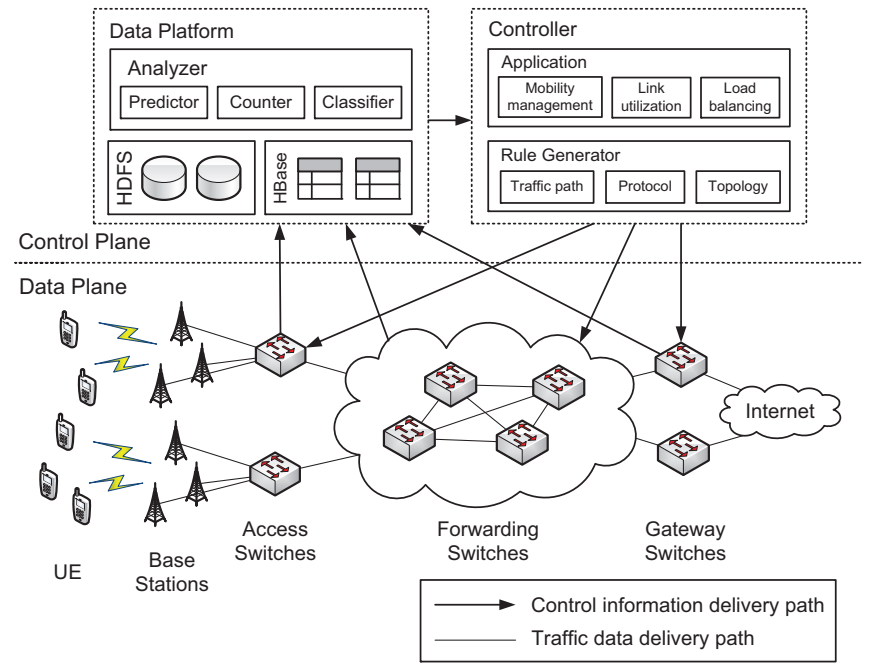

Fig. 1. The Big-Data-Enabled SDCN Architecture.

\section{A. Overview of the architecture}

Fig. 1 shows the design of BDEA. The network is decoupled into the control plane and the data plane. The control plane consists of the central controller and the network data storage and analysis platform, while the data plane corresponds to the data forwarding plane in traditional networks. Fig. 1 also shows how different kinds of data are delivered in the system. The links with arrows are the delivery paths of network information data and control messages. The solid lines (without arrows) indicate the forwarding paths of transmission data.

In the control plane, the controller manages the whole network with the cooperation of the data platform. The data storage and analysis platform stores the historical data, executes large-scale data analysis and offers different kinds of analysis results to the central controller. The detailed components in the control plane and the data exchanges among them will be introduced in the following section.

The data plane consists of base stations, access switches, forwarding switches and gateway switches. The user equipment (UE) links to the base stations through the wireless channels. A geographical cluster of base stations links to an access switch. The switches forward the data flows according to the flow tables, which are modified by the controller.

\section{B. Control plane design}

As Fig. 1 shows, in the control plane, different components cooperate with each other to implement network management. The data platform consists of mobile data storage and analysis components, while the controller includes the application layer and the rule generator.

In the data platform, Hadoop Distributed File System (HDFS) and Hadoop Database (Hbase) are chosen as they are among the best choices to store unstructured and structured data. The analyzers in the data platform include but are not limited to predictor, counter and classifier, which provide prediction results, statistical results and clustering results respectively for further resource allocation. 


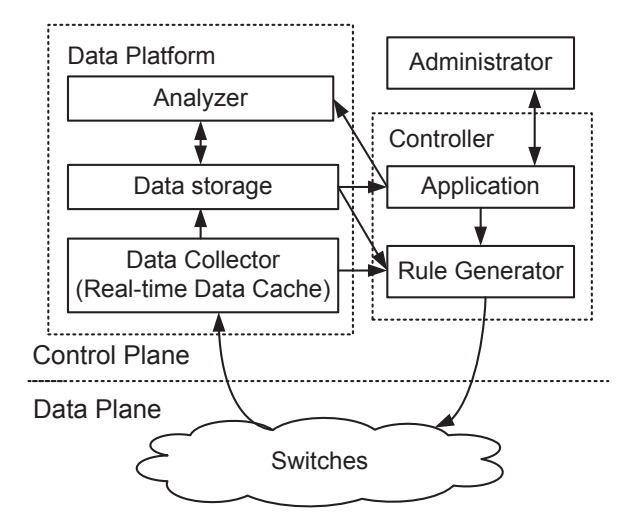

Fig. 2. The components and the data exchanges in the control plane.

In the controller, the application layer refers to the applications or algorithms which are designed to optimize the system performance in different aspects, such as mobility management, link utilization and load balancing. The rule generator conducts rules for the data plane according to the application layer and the analysis results.

Fig. 2 shows the components of the control plane and the data exchanges among them. In the following, we will introduce the functions and the relationships of these components in a network resource allocation procedure.

As the first step, the data collector extracts flow record frames and specific mirrored packets from the data plane. From these inputs, we can identify lots of information, such as user information (user ID, location, state), flow information (size, source, destination), service information (application type, service provider), network information (protocol type, link capacity, responding time), etc. As the collected information reflects the current status of the network, the data collector can be regarded as the real-time data cache.

For further usage, the collected information data will be forwarded to and stored in the data storage components. The files stored in HDFS can be parallel processed by MapReduce programs. The large and structured data set saved in HBase can be searched and updated with low latency.

In the next step, according to the request from applications, the analyzer in the data platform reads the data in the data storage component. By using different kinds of algorithms, the analyzer can generate various results, including but not limited to prediction results, statistical results and clustering results. These results will be written back into the data storage component for further usage and presentation.

Different kinds of results can offer different information to network administrators via the application layer. For example, statistical results, such as periodical traffic pattern and user profile can help network administrators understand network status and user groups more efficiently. Prediction results, such as user future movement and arriving traffic, can forecast network status in the future. Clustering results can group users, flows or services in different dimensions and improve the efficiency of algorithm deployment. Based on these analysis results, administrators can not only translate the data into business value, but also modify their network policies and

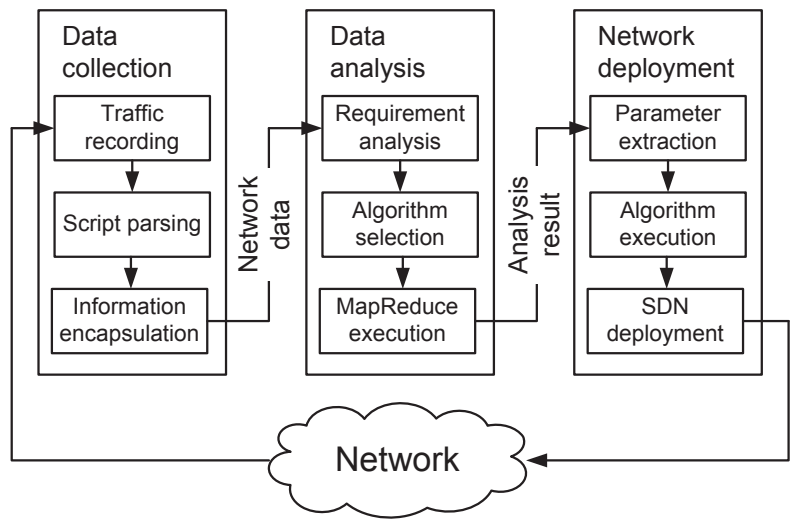

Fig. 3. The cycle of data collection, data analysis and network deployment.

applications to improve network performance.

In the controller, network administrators design applications and algorithms according to their demands. The rule generator conducts the network rules which are applied to the data plane directly. There are three inputs affecting decision making in the rule generator, namely the objectives and the constraints from the applications, the real-time network data from the data collector, and the analysis results generated and stored in the data platform. The parameters in the resource allocation algorithms can be modified dynamically, according to the analysis results, to achieve better performance. After the network rules are conducted, the controller applies them to the data plane via standard interfaces.

\section{The cycle of data collection, analysis and deployment}

Based on BDEA, we will present our design of the management cycle in this section. Fig. 3 shows the detailed procedure of the cycle. The three parts of the cycle, data collection, data analysis and network deployment, make up the cycle of network detection, development and deployment. The output of each part is the input of the following part.

In the data collection part, the system records the packets and flows in mobile networks and parse the scripts to extract network information. Then the data will be encapsulated in certain formats and stored for further analysis. According to the network administrators' requirements, in the data analysis part, the large-scale formatted data will be processed using selected algorithms. MapReduce would be used here as a tool to process big data. The analysis results then flow into the network deployment part, where it will be used for parameter extraction. The optimized parameter from the analysis results will be used in resource allocation algorithms. Finally, such resource allocation plans from these algorithms will be directly deployed to the network via the programmable interfaces.

The purpose of the cycle is to utilize big mobile data analysis outputs in advanced network resource allocation. With BDEA, the period of cellular network updating can be reduced and more data collected from the network can inform network management directly and quickly. 


\section{APPLICABILITY}

In this section, we will use several application cases to illustrate how the proposed cycle can use big mobile data analysis results to improve network performance in cellular network resource allocation. In these cases, prediction results, statistical results and clustering results from mobile data analysis are used for resource allocation, respectively.

\section{A. Mobility management}

Handover procedure is always a hot topic in mobility management in cellular communications. In traditional networks, MME makes handover decisions according to the measurement results of the user's reference signal received power (RSRP). This approach may lead to high delays, ping-pong effects and inefficient load balancing.

Taking the prediction results of big mobile data analysis into account, the handover decision making in BDEA can overcome the shortcomings in traditional handovers. According to the historical data and the current position of the mobile user, user movement prediction with high accuracy is feasible in big data analysis [5]. The controller can make handover decisions considering the predicted location and the current RSRP together. Data packets of the handover users can be prefetched to the predicted target base stations, thus reducing the handover delay caused by packet forwarding from the source base station to the target base station. Besides, by dynamically modifying the threshold of RSRP according to the observed ping-pong effects in the historical data, one can reduce the number of unnecessary handovers.

In the cases of overlapped macro, micro, pico and femto cells, the predicted location can lead to higher handover efficiency. The controller can select the target base station of different scales, according to the user's expected speed and location. The handover decision making with a global and predictive view can decrease the handover frequency, reduce the handover delay and balance the base station load.

\section{B. Energy and load management}

Based on the statistical results extracted from mass historical data, we can better understand the traffic volume in different areas in a day, a week, a month or a year. Traffic in mobile network presents periodical fluctuations in the temporal-spatial distribution [4] [10]. Traffic fluctuates over the day according to the mobile users' daily routine, as traffic fluctuates in commercial and residential areas according to people's work patterns. Such statistical results can be used to manage and operate switches and BSs in cellular networks.

In traditional networks, data packets are forwarded according to their forwarding rules stored in GWs. With BDEA, the rules of data forwarding can be set periodically as traffic fluctuates. Network operators can choose data forwarding plans for different periods over a day, turn off switches or BSs in low traffic periods or turn on network equipment beforehand to meet the future demands and overcome the peaks in the coming periods. The data forwarding plans matching traffic fluctuations can meet demands better, balance the load among $\mathrm{BSs}$ and reduce power consumption.

\section{Link resource allocation}

By mining users' attributes, mobile users can be clustered into different groups, according to their mobility patterns, application preference, traffic demands, etc. When predicting mobile users' locations, clustering the target users by their mobility patterns and choosing corresponding algorithms for each clusters can greatly improve the prediction accuracy [5]. Actually, the utilization of mobile user clustering can be extended to different resource allocation in cellular networks, such as in link resource allocation.

In BDEA, different link resource allocation plans can be designed for different user clusters, in order to improve users' experience. The controller can choose the paths for the packets according to the subscriber attributes and application types. The priority levels preset by network operators ensure that better service can be provided to certain user or application groups. When a new user joins the network, the data platform can analyze its attributes, categorize it into a certain group and advises a certain group of services.

\section{DISCUSSION}

In this section, we will compare the proposed management model with traditional approaches in some application cases. We will also discuss the challenges and the future work.

\section{A. Comparison with traditional cellular networks}

For comparison, we choose two application cases to illustrate that network performance can benefit from BDEA.

The first case is data prefetching in handovers. We use the prediction result of users' locations to prefetch data packets to possible target BSs before handover execution. The detailed algorithms and calculation process are presented in [11]. Fig. 4(a) and 4(b) show the comparison of the packet delays in the traditional and the BDEA approaches in handovers. The results show that when the packet arrival rate is low, the data prefetching strategy can decrease packet delay dramatically compared with traditional handovers. A satisfactory delay reduction is available with handover frequency fluctuations.

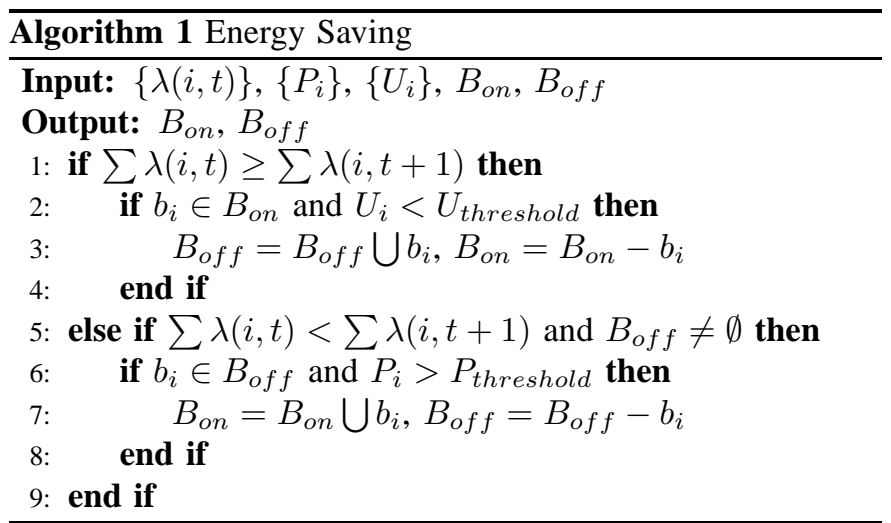

Alg. 1 shows an energy saving plan based on BS Sleeping. We firstly classify the BSs into clusters according to their locations and traffic patterns. Then we apply Alg. 1 to each cluster. $\lambda(i, t)$ is the arriving rate of data packets at the $i_{t h}$ BS in time slot $t$. The changing of $\lambda(i, t)$ shows the traffic 


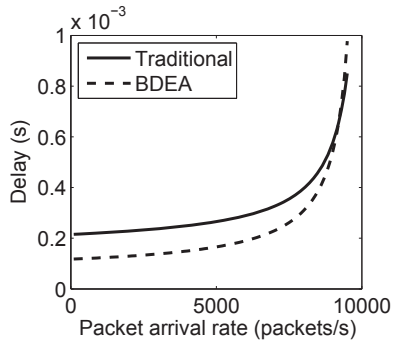

(a) Handover delay

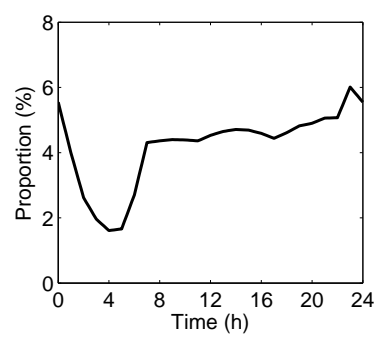

(c) Traffic volume over a day [10]

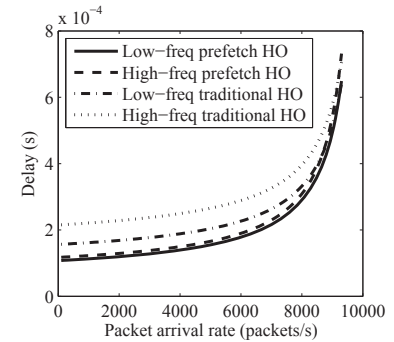

(b) Delay with different frequencies

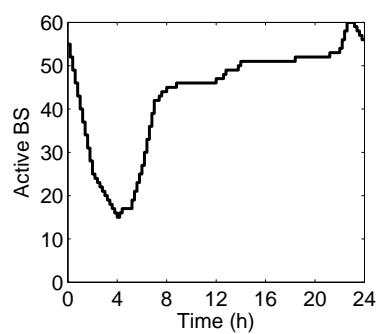

(d) Active BS in energy saving
Fig. 4. Comparison of the traditional and the BDEA approaches.

fluctuation trends. When $\lambda$ is increasing, the network turns on more BSs to satisfy the traffic demand. Otherwise, some BSs will get into sleep-mode for energy saving. Fig 4(d) shows the changing BS number according to the traffic volume over a day in Fig. 4(c). The algorithm tracks the traffic variation well despite the fluctuations. By reducing the number of active BSs in low traffic periods, cellular networks can reduce the total energy consumption. The detailed algorithms of this application will be improved and presented in our future work.

\section{B. Challenges}

Limited by the computing capacity in the separated devices in the existing cellular networks, the current resource allocation is implemented without the support from big data technology. BDEA provides an opportunity for resource allocation considering large-scale network data. However, the study on algorithms considering analysis results for dynamic resource allocation is still in its infancy. New research approaches and methodologies to utilize the big data analysis results need further study.

Besides, at the moment, the study on big mobile data analysis is mainly on user profile, which can be translated into business value for web service provider. The study benefitting network management, such as location and traffic prediction, is staying at the old level achieved several years ago without the support of big data analysis. Thus, new algorithms for network status analysis are needed with the development of big data analysis.

\section{Future work}

Considering the challenges above, in the future, we will mainly study resource allocation algorithms utilizing big mobile data analysis results. We will study the application cases listed in Section IV in details and develop new algorithms for resource allocation in cellular networks.
We will implement parts of BDEA separately and evaluate their performance by simulations. Data collected from real cellular networks will be used to implement the algorithms and test their performance.

\section{CONCLUSION}

In this paper, we propose a novel SDCN architecture BDEA which enables big data storage and analysis in cellular networks. The inter-component cooperation and the data exchange in the control plane make it feasible to assist network resource allocation with large-scale mobile data analysis. Based on BDEA, we also propose a cycle of data collection, data analysis and network deployment, which can increase the efficiency of network improvement and feedback.

To illustrate the advantages of BDEA, we study several application cases in which BDEA allows high efficiency in resource management. In these cases, prediction results, statistical results and clustering results from mobile data analysis are used for resource allocation. To compare with the traditional cellular networks, we give two application examples, namely, the data prefetching strategy and the energy saving plan. The results show that, benefiting from the analysis results, the packet delay in handovers and the energy consumption of BSs over a day can be substantially reduced. For future work, more algorithms for big mobile data analysis and utilization will be designed.

\section{REFERENCES}

[1] P. Cerwall et al., "Ericsson mobility report on the pules of the networked society," Ericsson AB, Stockholm, Sweden, Tech. Rep., Nov 2015.

[2] J. Liu, F. Liu, and N. Ansari, "Monitoring and analyzing big traffic data of a large-scale cellular network with hadoop," IEEE Network, vol. 28, no. 4, pp. 32-39, July 2014.

[3] Y. Lee and Y. Lee, "Toward scalable internet traffic measurement and analysis with hadoop," SIGCOMM Computer Communication Review, vol. 43, no. 1, pp. 5-13, Jan 2012.

[4] J. Yang, Y. Qiao, X. Zhang, H. He, F. Liu, and G. Cheng, "Characterizing user behavior in mobile internet," IEEE Transactions on Emerging Topics in Computing, vol. 3, no. 1, pp. 95-106, March 2015.

[5] H. He, Y. Qiao, S. Gao, J. Yang, and J. Guo, "Prediction of user mobility pattern on a network traffic analysis platform," in Proc. 10th International Workshop on Mobility in the Evolving Internet Architecture, Paris, France, Sep 2015, pp. 39-44.

[6] L. E. Li, Z. M. Mao, and J. Rexford, "Toward software-defined cellular networks," in Proc. IEEE 2012 European Workshop on Software Defined Networking (EWSDN), Darmstadt, Germany, Oct 2012, pp. 7-12.

[7] A. Gudipati, D. Perry, L. E. Li, and S. Katti, "SoftRAN: Software defined radio access network," in Proc. 2nd ACM SIGCOMM Workshop on Hot Topics in Software Defined Networking, Hong Kong, China, 2013, pp. 25-30.

[8] X. Jin, L. E. Li, L. Vanbever, and J. Rexford, "SoftCell: Scalable and flexible cellular core network architecture," in Proc. 9th ACM Conference on Emerging Networking Experiments and Technologies, ser. CoNEXT '13, Santa Barbara, USA, 2013, pp. 163-174.

[9] M. Moradi, W. Wu, L. E. Li, and Z. M. Mao, "SoftMoW: Recursive and reconfigurable cellular WAN architecture," in Proc. 10th ACM International Conference on Emerging Networking Experiments and Technologies, ser. CoNEXT' 14, 2014, pp. 377-390.

[10] J. Yang, H. He, and Y. Qiao, "Network traffic analysis based on Hadoop," in Proc. 4th International Conference on Wireless Communications, Vehicular Technology, Information Theory and Aerospace Electronic Systems (VITAE), Aalborg, Denmark, May 2014, pp. 1-5.

[11] J. Wen and V. O. K. Li, "Data prefetching to reduce delay in SoftwareDefined cellular networks," in Proc. IEEE 26th International Symposium on Personal, Indoor and Mobile Radio Communications, Hong Kong, Aug 2015, pp. 1845-1849. 